\title{
Quasi-valuations - topology and the weak approximation theorem
}

\author{
Shai Sarussi
}

\begin{abstract}
Suppose $F$ is a field with a nontrivial valuation $v$ and valuation ring $O_{v}, E$ is a finite field extension and $w$ is a quasi-valuation on $E$ extending $v$. We study the topology induced by $w$. We prove that the quasi-valuation ring determines the topology, independent of the choice of its quasi-valuation. Moreover, we prove the weak approximation theorem for quasi-valuations.
\end{abstract}

\section{Introduction}

Recall that a valuation on a field $F$ is a function $v: F \rightarrow \Gamma \cup\{\infty\}$, where $\Gamma$ is a totally ordered abelian group and $v$ satisfies the following conditions:

(A1) $v(x)=\infty$ iff $x=0$;

(A2) $v(x y)=v(x)+v(y)$ for all $x, y \in F$;

(A3) $v(x+y) \geq \min \{v(x), v(y)\}$ for all $x, y \in F$.

There has been considerable interest in recent years in generalizations of valuations, in order to treat rings that are not integral domains, and also to handle several valuations simultaneously. For example, pseudo-valuations (see $[\mathrm{Co}],[\mathrm{Hu}]$, and $[\mathrm{MH}]$ ), Manis-valuations and PM-valuations (see [KZ]), value functions (see [Mor]), and gauges (see [TW]). These related theories are discussed briefly in the introduction of [Sa].

In this paper we continue our study from [Sa] of quasi-valuations. Recall that a quasi-valuation on a ring $A$ is a function $w: A \rightarrow M \cup\{\infty\}$, where $M$ is a totally ordered abelian monoid, to which we adjoin an element $\infty$ greater than all elements of $M$, and $w$ satisfies the following properties:

(B1) $w(0)=\infty$;

(B2) $w(x y) \geq w(x)+w(y)$ for all $x, y \in A$;

(B3) $w(x+y) \geq \min \{w(x), w(y)\}$ for all $x, y \in A$.

The minimum of a finite number of valuations with the same value group is a quasi-valuation. For example, the $n$-adic quasi-valuation on $\mathbb{Q}$ (for any positive $n \in \mathbb{Z}$ ) already has been studied in [Ste]. (Stein calls it the $n$-adic valuation.) It is defined as follows: for any $0 \neq \frac{c}{d} \in \mathbb{Q}$ there exists a unique $e \in \mathbb{Z}$ and integers $a, b \in \mathbb{Z}$, with $b$ positive, such that $\frac{c}{d}=n^{e} \frac{a}{b}$ with $n \nmid a$, $(n, b)=1$ and $(a, b)=1$. Define $w_{n}\left(\frac{c}{d}\right)=e$ and $w_{n}(0)=\infty$. 
In [Sa] we develop the theory of quasi-valuations on finite dimensional field extensions that extend a given valuation. For the reader's convenience we briefly overview some of the results from [Sa]. Let $F$ be a field with valuation $v$ and valuation ring $O_{v}$, let $E$ be a finite field extension and let $w$ be a quasi-valuation on $E$ extending $v$ with a corresponding quasi-valuation ring $O_{w}$. We prove that $O_{w}$ satisfies INC (incomparability), LO (lying over), and GD (going down) over $O_{v}$; in particular, $O_{w}$ and $O_{v}$ have the same Krull Dimension. We also prove that every such quasi-valuation is dominated by some valuation extending $v$. Namely, there exists a valuation $u$ extending $v$ on $E$ so that $\forall x \in E, w(x) \leq u(x)$.

Under the assumption that the value monoid of the quasi-valuation is a group we prove that $O_{w}$ satisfies GU (going up) over $O_{v}$, and a bound on the size of the prime spectrum is given. In addition, a 1:1 correspondence is obtained between exponential quasi-valuations and integrally closed quasivaluation rings.

Given $R$, an algebra over $O_{v}$, we construct a quasi-valuation on $R$; we also construct a quasi-valuation on $R \otimes_{O_{v}} F$, which helps us prove our main Theorem. The main Theorem states that if $R \subseteq E$ satisfies $R \cap F=O_{v}$ and $E$ is the field of fractions of $R$, then $R$ and $v$ induce a quasi-valuation $w$ on $E$ such that $R=O_{w}$ and $w$ extends $v$; thus $R$ satisfies the properties of a quasi-valuation ring.

In this paper, we extend some fundamental results from valuation theory. For example, we prove that the topology induced by a quasi-valuation is Hausdorff and totally disconnected. We also prove a weak version of the approximation theorem for quasi-valuations.

\section{The topology induced by a quasi-valuation}

In this section we introduce the topology induced by a quasi-valuation. We show that this topology is close to the topology induced by a valuation in the sense that they share some basic topological properties such as being both Hausdorff and totally disconnected. In the main theorem of this section we prove that the topology induced by the quasi-valuation is determined by the corresponding quasi-valuation ring.

In this section $F$ denotes a field with a nontrivial valuation $v$, a value group $\Gamma$, and a valuation ring $O_{v} . E$ denotes a finite dimensional field extension with $n=[E: F], w: E \rightarrow M \cup\{\infty\}$ is a quasi-valuation on $E$ with quasi-valuation ring $O_{w}$ (namely, $O_{w}=\{x \in E \mid w(x) \geq 0\}$ ), such that $\left.w\right|_{F}=v$, and $M$ is a totally ordered abelian monoid containing $\Gamma$.

We note that $\mathrm{w}(-1)=0$ because $w$ extends $v$. Thus, by [Sa, Lemma 1.3], we have $w(x)=w(-x)$ for all $x \in E$. Moreover, by [Sa, Lemma 1.4], for all $x, y \in E$ such that $w(x) \neq w(y)$, we have $w(x+y)=\min \{w(x), w(y)\}$. Recall from [Sa, Definition 1.5] that an element $c \in E$ is called stable with 
respect to $w$ if $w(c x)=w(c)+w(x)$ for every $x \in E$. Thus, by [Sa, Lemma 1.6], every $a \in F$ is stable with respect to $w$. We shall freely use these facts throughout the paper.

Let $x \in E$ and $m \in M$; we denote

$$
U_{m}^{w}(x)=\{y \in E \mid w(y-x)>m\} ;
$$

we suppress $w$ when it is understood.

Remark 2.1. Let $x \in E$ and $m \in M$; then $x \in U_{m}(x)$. Indeed,

$$
w(x-x)=w(0)=\infty>m .
$$

We shall repeatedly use Remark 2.1 without reference.

Lemma 2.2. If $y \in U_{m_{1}}\left(x_{1}\right) \cap U_{m_{2}}\left(x_{2}\right)$ and $m_{1} \leq m_{2}$, then

$$
y \in U_{m_{2}}(y) \subseteq U_{m_{1}}\left(x_{1}\right) \cap U_{m_{2}}\left(x_{2}\right) .
$$

Proof. Since $y \in U_{m_{1}}\left(x_{1}\right) \cap U_{m_{2}}\left(x_{2}\right)$, we have $w\left(y-x_{1}\right)>m_{1}$ and $w\left(y-x_{2}\right)>m_{2} \geq m_{1}$. Let $z \in U_{m_{2}}(y)$; then $w(z-y)>m_{2}$. Thus,

$$
\begin{gathered}
w\left(z-x_{1}\right)=w\left(z-y+y-x_{1}\right) \\
\geq \min \left\{w(z-y), w\left(y-x_{1}\right)\right\}>m_{1}
\end{gathered}
$$

and

$$
\begin{gathered}
w\left(z-x_{2}\right)=w\left(z-y+y-x_{2}\right) \\
\geq \min \left\{w(z-y), w\left(y-x_{2}\right)\right\}>m_{2} .
\end{gathered}
$$

Thus $z \in U_{m_{1}}\left(x_{1}\right) \cap U_{m_{2}}\left(x_{2}\right)$

We denote $B=\left\{U_{m}(x) \mid x \in E, m \in M\right\}$.

Corollary 2.3. The set $B$ is a base for a topology on $E$.

In view of Corollary 2.3 we define,

Definition 2.4. The topology whose base is $B$ will be denoted by $T_{w}$. We call $T_{w}$ the topology induced by the quasi-valuation $w$.

We recall the following lemma from [Sa, Lemma 2.8]:

Lemma 2.5. Let $E / F$ be a finite field extension and let $w$ be a quasivaluation on $E$ extending a valuation $v$ on $F$. Then $w(x) \neq \infty$ for all $0 \neq x \in E$. In fact, for all $0 \neq x \in E$, there exists $\alpha \in \Gamma$ such that $w(x)<\alpha$. 
We denote

$$
M^{G}=\{m \in M \mid \text { there exists } 0 \neq y \in E \text { such that } m \leq w(y)\} .
$$

Remark 2.6. $M^{G}$ is a submonoid of $M$ containing $\Gamma$.

Proof. Let $m_{1}, m_{2} \in M^{G}$; then there exist nonzero $y_{1}, y_{2} \in E$ such that $m_{1} \leq w\left(y_{1}\right)$ and $m_{2} \leq w\left(y_{2}\right)$. Thus,

$$
m_{1}+m_{2} \leq w\left(y_{1}\right)+w\left(y_{2}\right) \leq w\left(y_{1} y_{2}\right) .
$$

Note that $y_{1} y_{2} \neq 0$ and thus $m_{1}+m_{2} \in M^{G}$. It is easy to see that $\Gamma \subseteq M^{G}$; indeed, for every $\alpha \in \Gamma$ there exists a nonzero $a \in F$ such that $w(a)=$ $v(a)=\alpha$.

Remark 2.7. $M^{G}$ does not have a maximal element; in fact, for all $m \in M^{G}$ there exists $\alpha \in \Gamma \cap M^{G}$ such that $m<\alpha$.

Proof. Let $m \in M^{G}$. Then there exists $0 \neq y \in E$ such that $m \leq w(y)$. By Lemma 2.5 there exists $\alpha \in \Gamma$ such that $w(y)<\alpha$. So, $m<\alpha$. By Remark 2.6. $\alpha \in \Gamma \subseteq M^{G}$.

Proposition 2.8. $T_{w}$ is discrete iff there exists an element $m \in M \backslash M^{G}$.

Proof. $(\Leftarrow)$ Let $m \in M \backslash M^{G}$ and let $x \in E$. Then for every $y \neq x$ we have $w(y-x)<m$; thus $U_{m}(x)=\{x\}$.

$(\Rightarrow)$ We assume $M=M^{G}$ and we show that $T_{w}$ is not discrete. It is enough to show that every open set has infinitely many elements. Now, since every open set contains some $U_{m}(x)$ (for $m \in M, x \in E$ ), it is enough to show that every $U_{m}(x)$ has infinitely many elements. By our assumption $M=M^{G}$ and thus for every $m \in M$ there exists $0 \neq z \in E$ such that $m \leq w(z)$; also, by Lemma 2.5, for every such $z$ there exists $0<\alpha \in \Gamma$ such that $w(z)<\alpha$. Take $a \in O_{v}$ with $v(a)=\alpha$. Then $x+a^{n} \in U_{m}(x)$ for each $n \in \mathbb{N}$, proving the set $U_{m}(x)$ has infinitely many elements.

In view of Proposition 2.8, we restrict our discussion to $M^{G}$; namely we denote $B=\left\{U_{m}(x) \mid x \in E, m \in M^{G}\right\}$ as a base for $T_{w}$.

Lemma 2.9. Let $x, y, z \in E$ and $m \in M^{G}$. If $z \in U_{m}(x) \cap U_{m}(y)$ then $w(y-x)>m$.

Proof. By definition, $z \in U_{m}(x) \cap U_{m}(y)$ implies $w(z-x)>m$ and $w(y-z)>$ $m$. Thus,

$$
w(y-x) \geq \min \{w(y-z), w(z-x)\}>m .
$$


Proposition 2.10. $T_{w}$ is Hausdorff.

Proof. Let $x, y \in E$ with $x \neq y$, and write $w(y-x)=m \in M^{G}$. By Lemma 2.9 we have,

$$
U_{m}(x) \cap U_{m}(y)=\emptyset .
$$

Lemma 2.11. Let $x \in E$ and $m \in M^{G}$. Then $U_{m}(x)$ is closed as well as open.

Proof. Let $y \notin U_{m}(x)$; then $w(y-x) \leq m$. By Lemma 2.9 we have,

$$
U_{m}(y) \cap U_{m}(x)=\emptyset
$$

obviously $y \in U_{m}(y)$. So, $U_{m}(y)$ is an open set containing $y$ disjoint from $U_{m}(x)$.

The following lemma shows that $E$ is totally disconnected, in the following sense.

Proposition 2.12. The only nonempty connected subsets of $E$ are the singleton sets $\{x\}$ for $x \in E$.

Proof. Let $S \subseteq E$ be a nonempty set containing at least two elements, $x \neq y$. Write $w(x-y)=m$ and $U_{1}=U_{m}(x)$. Let $U_{2}$ denote the complement of $U_{1}$ in $E$, which is open by Lemma 2.11. Note that $x \in U_{1}$ and $y \in U_{2}$ (since $w(y-x)=m \ngtr m)$, and thus by definition $S$ is disconnected.

Let $x \in E$ and $m \in M^{G}$; we denote $\widetilde{U}_{m}(x)=\{y \in E \mid w(y-x) \geq m\}$. Obviously, $U_{m}(x) \subseteq \widetilde{U}_{m}(x)$. Thus, as in Remark 2.1, we have $x \in \widetilde{U}_{m}(x)$.

Lemma 2.13. Let $x, y \in E$ and $m \in M^{G}$. If $y \in \widetilde{U}_{m}(x)$ then $\widetilde{U}_{m}(y) \subseteq$ $\widetilde{U}_{m}(x)$. If $y \notin \widetilde{U}_{m}(x)$ then $\widetilde{U}_{m}(y) \subseteq\left(\widetilde{U}_{m}(x)\right)^{c}$.

Proof. Suppose $y \in \widetilde{U}_{m}(x)$; then $w(y-x) \geq m$. Let $z \in \widetilde{U}_{m}(y)$; then $w(z-y) \geq m$. Hence, $w(z-x) \geq \min \{w(z-y), w(y-x)\} \geq m$. Thus,

$$
\widetilde{U}_{m}(y) \subseteq \widetilde{U}_{m}(x) .
$$

Suppose $y \notin \widetilde{U}_{m}(x)$; then $w(y-x)<m$. Let $z \in \widetilde{U}_{m}(y)$; then $w(z-y) \geq$ $m$. Hence, $w(z-x)=\min \{w(z-y), w(y-x)\}=w(y-x)<m$. Thus,

$$
\widetilde{U}_{m}(y) \subseteq\left(\widetilde{U}_{m}(x)\right)^{c} .
$$


Corollary 2.14. $\widetilde{U}_{m}(x)$ is both open and closed, for any $x \in E$ and $m \in$ $M^{G}$.

Proof. Let $y \in \widetilde{U}_{m}(x)$; then by Lemma 2.13 ,

$$
y \in U_{m}(y) \subseteq \widetilde{U}_{m}(y) \subseteq \widetilde{U}_{m}(x)
$$

Let $y \notin \widetilde{U}_{m}(x)$; then by Lemma 2.13 ,

$$
y \in U_{m}(y) \subseteq \widetilde{U}_{m}(y) \subseteq\left(\widetilde{U}_{m}(x)\right)^{c} .
$$

Lemma 2.15. Let $x \in E$ and let $m, m^{\prime} \in M^{G}$ such that $m<m^{\prime}$. Then

$$
U_{m}(x)=\bigcup_{y \in U_{m}(x)} \widetilde{U}_{m^{\prime}}(y)
$$

Proof. ( $\subseteq$ ) holds because every $y \in U_{m}(x)$ is obviously in $\widetilde{U}_{m^{\prime}}(y)$. To prove $(\supseteq)$, we need to show that $\widetilde{U}_{m^{\prime}}(y) \subseteq U_{m}(x)$ for all $y \in U_{m}(x)$. So, let $y \in U_{m}(x)$ and let $z \in \widetilde{U}_{m^{\prime}}(y)$. Then, $w(y-x)>m$ and $w(z-y) \geq m^{\prime}$. Thus, since $m<m^{\prime}$,

$$
w(z-x) \geq \min \{w(z-y), w(y-x)\}>m .
$$

We denote $B_{1}=\left\{\widetilde{U}_{m}(x) \mid x \in E, m \in M^{G}\right\}$.

Proposition 2.16. The set $B_{1}$ is a base for $T_{w}$.

Proof. First, by Corollary 2.14, $\widetilde{U}_{m}(x)$ is open for all $x \in E$ and $m \in M^{G}$. Now, let $x \in E$ and $m \in M^{G}$. By Remark 2.7 there exists $m<m^{\prime} \in M^{G}$. By Lemma 2.15 we have, $U_{m}(x)=\bigcup_{y \in U_{m}(x)} \widetilde{U}_{m^{\prime}}(y)$. Thus, every open set in $T_{w}$ is a union of elements of $B_{1}$.

In fact, we can describe the topology in terms of $\Gamma$ (the value group of the valuation) as the following proposition shows.

First, we denote $B_{2}=\left\{\widetilde{U}_{\alpha}(x) \mid x \in E, \alpha \in \Gamma\right\}$.

Proposition 2.17. The set $B_{2}$ is a base for $T_{w}$.

Proof. First, by Remark 2.6, $B_{2} \subseteq B_{1}$. So every element of $B_{2}$ is open in $T_{w}$. Now, let $x \in E$ and $m \in M^{G}$. By Remark 2.7, there exists $\alpha \in \Gamma \cap M^{G}$ such that $m<\alpha$. By Lemma 2.15,

$$
U_{m}(x)=\bigcup_{y \in U_{m}(x)} \widetilde{U}_{\alpha}(y) .
$$


Recall from [Sa, Section 10] that for every ring $R \subseteq E$ satisfying $R \cap F=$ $O_{v}$, we denote

$\mathcal{W}_{R}=\left\{w \mid w\right.$ is a quasi-valuation on $E$ extending $v$ with $\left.O_{w}=R\right\}$.

Also recall that the class $\mathcal{W}_{R}$ is not empty, by [Sa, Theorem 9.35].

Lemma 2.18. Let $w \in \mathcal{W}_{R}, x \in E$ and $0 \neq a \in F$. The following are equivalent:
(a) $w(x) \geq v(a)$
(b) $w(x)-v(a) \geq 0$;
(c) $w\left(x a^{-1}\right) \geq 0$;
(d) $x a^{-1} \in R$.

Proof. (a) $\Leftrightarrow(\mathrm{b})$. Because $v(a) \in \Gamma$. (b) $\Leftrightarrow(\mathrm{c}) . v$ is a valuation and $0 \neq a \in F$; thus $-v(a)=v\left(a^{-1}\right)$. Therefore, $w(x)-v(a)=w(x)+v\left(a^{-1}\right)$. Since $w$ extends $v$ and $a$ is stable with respect to $w$, we get

$$
w(x)+v\left(a^{-1}\right)=w(x)+w\left(a^{-1}\right)=w\left(x a^{-1}\right) .
$$

(c) $\Leftrightarrow\left(\right.$ d). By assumption, $w \in \mathcal{W}_{R}$; thus $O_{w}=R$.

Lemma 2.19. Let $w_{1}, w_{2} \in \mathcal{W}_{R}$ and let $\alpha \in \Gamma$; then

$$
w_{1}(x) \geq \alpha \text { iff } w_{2}(x) \geq \alpha .
$$

Proof. By assumption, $O_{w_{1}}=O_{w_{2}}=R$. Let $a \in F$ such that $v(a)=\alpha$; clearly, $a \neq 0$ (since $\alpha \in \Gamma$ ). Thus, using Lemma 2.18 twice, we get

$$
w_{1}(x) \geq \alpha \text { iff } x a^{-1} \in O_{w_{1}}=O_{w_{2}} \text { iff } w_{2}(x) \geq \alpha .
$$

We are ready to prove the main theorem of this section.

Theorem 2.20. If $w_{1}, w_{2} \in \mathcal{W}_{R}$, then $T_{w_{1}}=T_{w_{2}}$. In other words, the quasi-valuation ring determines the topology, independent of the choice of its quasi-valuation.

Proof. By Proposition 2.17, the set $C=\left\{\widetilde{U}_{\alpha}^{w_{1}}(x) \mid x \in E, \alpha \in \Gamma\right\}$ is a base for $T_{w_{1}}$ and the set $D=\left\{\widetilde{U}_{\alpha}^{w_{2}}(x) \mid x \in E, \alpha \in \Gamma\right\}$ is a base for $T_{w_{2}}$. However, for every $x \in E$ and $\alpha \in \Gamma$ we have, by Lemma 2.19, $\widetilde{U}_{\alpha}^{w_{1}}(x)=\widetilde{U}_{\alpha}^{w_{2}}(x)$. Thus, $C=D$ and the theorem is proved. 


\section{$3 \quad$ Weak approximation theorem}

In this section we prove a weak version of the approximation theorem for quasi-valuations. We call it the weak approximation theorem since it relies on the independence of the valuation rings in $F$ and not on the independence of the quasi-valuations in $E$. (The independence of the valuation rings in $F$ implies the independence of the quasi-valuation rings in $E$ but not vice versa).

In this section $F$ denotes a field and $E$ denotes a finite dimensional field extension with $[E: F]=n$.

Definition 3.1. Let $A$ and $B$ be two subrings of $F$. $A$ and $B$ are called independent if $A B=F$. Two valuations are called independent, if their rings are independent; likewise, two valuations are called dependent, if their rings are dependent.

We recall the Approximation Theorem for valuations.

Theorem 3.2. (Approximation Theorem for valuations) ([Bo, Section 7.2, Thm. 1]) Let $\left\{v_{i}\right\}_{1 \leq i \leq k}$ be a set of valuations on a field $F$ which are independent in pairs and let $\Gamma_{i}$ be the value group of $v_{i}$. Let $x_{i} \in F$ and $\alpha_{i} \in \Gamma_{i}$ for $1 \leq i \leq k$. Then there exists an $x \in F$ such that $v_{i}\left(x-x_{i}\right) \geq \alpha_{i}$ for all $i$.

Let $\left\{O_{v_{i}}\right\}_{1 \leq i \leq k}$ be a finite set of valuation rings of $F$. We denote by $B$ their intersection, i.e.,

$$
B=\bigcap_{1 \leq i \leq k} O_{v_{i}} .
$$

Let $\left\{R_{i}\right\}_{1 \leq i \leq k}$ be a finite set of subrings of $E$ such that $E$ is the field of fractions of each $R_{i}$ and $R_{i} \cap F=O_{v_{i}}$ for every $1 \leq i \leq k$. Recall that by [Sa, Theorem 9.35], for every $1 \leq i \leq k$ there exists a filter quasi-valuation $w_{i}$ on $E$ corresponding to $R_{i}$ and such that $w_{i}$ extends $v_{i}$ (so the collections of quasi-valuations corresponding to the $R_{i}$ 's are not empty.) We shall prove our theorem for every quasi-valuation $w_{i}$ on $E$ corresponding to $R_{i}$ (not necessarily filter quasi-valuations). Note that for every $1 \leq i \leq k, R_{i} F$ is an integral domain finite dimensional over $F$ and thus a field containing $R_{i}$; hence $R_{i} F=E$. Moreover, by [Bo, Section 7, Proposition 1], the field of fractions of $B$ is $F$.

We denote $R=\bigcap_{1 \leq i \leq k} R_{i}$.

The next observation is well known.

Remark 3.3. Let $C$ be an integral domain, $S$ a multiplicative closed subset of $C$ with $0 \notin S$, and $R$ an algebra over $C$. We claim that every $x \in R \otimes_{C} C S^{-1}$ is of the form $r \otimes \frac{1}{\beta}$ for $r \in R$ and $\beta \in S$. Indeed, write $x=\sum_{i=1}^{t}\left(r_{i} \otimes \frac{\alpha_{i}}{\beta_{i}}\right)$ where $r_{i} \in R, \alpha_{i} \in C$ and $\beta_{i} \in S$. Let $\beta=\Pi_{i=1}^{t} \beta_{i}$ and $\alpha_{i}^{\prime}=\alpha_{i} \beta \beta_{i}^{-1} \in C$. 
Thus,

$$
\sum_{i=1}^{t}\left(r_{i} \otimes \frac{\alpha_{i}}{\beta_{i}}\right)=\sum_{i=1}^{t}\left(r_{i} \otimes \frac{\alpha_{i}^{\prime}}{\beta}\right)=\sum_{i=1}^{t}\left(\alpha_{i}^{\prime} r_{i} \otimes \frac{1}{\beta}\right)=r \otimes \frac{1}{\beta} .
$$

Where $r=\sum_{i=1}^{t} \alpha_{i}^{\prime} r_{i}$.

Proposition 3.4. $E=S^{-1} R \cong R \otimes_{B} F$, where $S=B \backslash\{0\}$.

Proof. $S^{-1} R$ is an integral domain finite dimensional over $F$, so is a field. It remains to show that any $x \in E$ has the form $r / b$ where $r \in R$ and $b \in S$. By the previous Remark, $x$ can be written in the form $r_{i} / b_{i}$ where $r_{i} \in R_{i}$ and $b_{i} \in B \backslash\{0\}$. Write $b=\prod_{1 \leq i \leq k} b_{i}$ and get

$$
b x=r_{i} \prod_{l \neq i} b_{l} \in R_{i} B=R_{i}
$$

for every $1 \leq i \leq k$, and thus $x=b x / b$ has the desired form.

We are ready for the main theorem of this section: the weak approximation theorem for quasi-valuations.

Theorem 3.5. Let $E / F$ be a finite field extension and let $\left\{O_{v_{i}}\right\}_{1 \leq i \leq k}$ be a finite set of valuation rings of $F$ which are pairwise independent. Let $\left\{R_{i}\right\}_{1 \leq i \leq k}$ be a finite set of subrings of $E$ such that the field of fractions of each $R_{i}$ is $E$ and $R_{i} \cap F=O_{v_{i}}$ for every $1 \leq i \leq k$. Let $\left\{w_{i} \mid w_{i}: E \rightarrow\right.$ $\left.M_{i} \cup\{\infty\}, 1 \leq i \leq k\right\}$ be a set of quasi-valuations on $E$ such that for every $1 \leq i \leq k, w_{i} \in \mathcal{W}_{R_{i}}$. Let $\left\{x_{i}\right\}_{1 \leq i \leq k} \subseteq E$ and let $\left\{m_{i}\right\}_{1 \leq i \leq k}$ be a set of elements such that, for every $1 \leq i \leq k, m_{i} \in M_{i}^{G}$. Then there exists an element $x \in E$ such that

$$
w_{i}\left(x-x_{i}\right) \geq m_{i}
$$

for all $1 \leq i \leq k$.

Proof. Since $m_{i} \in M_{i}^{G}$ for every $1 \leq i \leq k$, we get by Remark 2.7 that there exist $\alpha_{i} \in \Gamma_{i}$ such that $m_{i}<\alpha_{i}$ for all $1 \leq i \leq k$. We shall prove that $w_{i}\left(x-x_{i}\right) \geq \alpha_{i}$ for every $1 \leq i \leq k$. Let $R=\bigcap_{1<i<k} R_{i}$; by Proposition 3.4, $R$ contains a basis $\left\{r_{1}, r_{2}, \ldots, r_{n}\right\}$ of $E$ over $F$. Write, for every $1 \leq i \leq k$,

$$
x_{i}=\sum_{1 \leq j \leq n} c_{i j} r_{j}
$$

where $c_{i j} \in F$. The approximation theorem for valuations gives $d_{1}, \ldots, d_{n} \in F$ such that

$$
v_{i}\left(d_{j}-c_{i j}\right) \geq \alpha_{i}
$$

for $1 \leq i \leq k, 1 \leq j \leq n$. 
Define $x=\sum_{1 \leq j \leq n} d_{j} r_{j}$ and get, for every $1 \leq i \leq k$,

$$
\begin{gathered}
w_{i}\left(x-x_{i}\right)=w_{i}\left(\sum_{1 \leq j \leq n} d_{j} r_{j}-\sum_{1 \leq j \leq n} c_{i j} r_{j}\right) \\
=w_{i}\left(\sum_{1 \leq j \leq n}\left(d_{j}-c_{i j}\right) r_{j}\right) .
\end{gathered}
$$

Note that, for every $1 \leq j \leq n$ and $1 \leq i \leq k$,

$$
w_{i}\left(d_{j}-c_{i j}\right)=v_{i}\left(d_{j}-c_{i j}\right) \geq \alpha_{i}
$$

and $w_{i}\left(r_{j}\right) \geq 0$ (since $\left.r_{j} \in R\right)$. Thus,

$$
w_{i}\left(\sum_{1 \leq j \leq n}\left(d_{j}-c_{i j}\right) r_{j}\right) \geq \min _{1 \leq j \leq n} w_{i}\left(\left(d_{j}-c_{i j}\right) r_{j}\right) \geq \alpha_{i} .
$$

\section{References}

[Bo] N. Bourbaki, Commutative Algebra, Chapter 6, Valuations, Hermann, Paris, 1961.

[Co] P. M. Cohn, An Invariant Characterization of Pseudo-Valuations, Proc. Camp. Phil. Soc. 50 (1954), 159-177.

[End] O. Endler, Valuation Theory, Springer-Verlag, New York, 1972.

[Hu] J. A. Huckaba, Extensions of Pseudo-Valuations, Pacific J. Math. Volume 29, Number 2 (1969), 295-302.

[KZ] M. Knebusch and D. Zhang, Manis Valuations and Prüfer Extensions, Springer-Verlag, Berlin, 2002.

[MH] M. Mahadavi-Hezavehi, Matrix Pseudo-Valuations on Rings and their Associated Skew Fields, Int. Math. J. 2 (2002), no. 1, 7-30.

[Mor] P. J. Morandi, Value functions on central simple algebras, Trans. Amer. Math. Soc., 315 (1989), 605-622.

[Sa] S. Sarussi, Quasi-valuations extending a valuation, J. Algebra 372 (2012), 318-364.

[TW] J.-P. Tignol and A.R. Wadsworth, Value functions and associated graded rimgs for semisimple algebras, Trans. Amer. Math. Soc., 362 (2010), 687-726. 
[Wad] A.R. Wadsworth, Valuation theory on finite dimensional division algebras, pp. 385-449 in Valuation Theory and its Applications, Vol. 1, eds. F.-V. Kuhlmann et al., Fields Inst. Commun., 32, American Mathematical Society, Providence, RI, 2002.

Department of Mathematics, Sce College, Ashdod, 77245, Israel.

E-mail address: sarusss1@gmail.com 